\title{
Operational and Clinical Implementation Plan for an Anesthesiologist-Led Coronavirus-2019 Hospital "SWAT" Team
}

\author{
Jammie T Ferrara ${ }^{1}$, Richard Y Kim ${ }^{1}$, Jan M Gruber ${ }^{1}$, Katherine R Arthur ${ }^{1}$, Edward Y Chen ${ }^{1}$, Dhilan A Thuraisingham ${ }^{1}$, Melissa \\ Trelatsky CRNA ${ }^{1}$, Sanna J Lee ${ }^{1}$, Nichole Donahue ${ }^{1}$, Arash Kharestan ${ }^{2}$, Luis M Moreta-Sainz ${ }^{2}$, Danielle S Stranc ${ }^{1}$, Andrew G \\ Rudikoff $^{1}$ and Antonio Hernandez Conte ${ }^{1 *}$ \\ ${ }^{1}$ Department of Anesthesiology, Kaiser Permanente Los Angeles Medical Center, 4867 Sunset Blvd. Los Angeles, California 90027, USA \\ ${ }^{2}$ Department of Internal Medicine, Division of Pulmonary Medicine, Kaiser Permanente Los Angeles Medical Center, 4867 Sunset Blvd. Los Angeles, \\ California 90027, USA
}

${ }^{\star}$ Corresponding author: Antonio Hernandez Conte, Department of Anesthesiology, Kaiser Permanente Los Angeles Medical Center, 4867 Sunset Blvd. Los Angeles, California 90027, USA; Phone: 323 3238746; Email: Antonio.Conte@kp.org

Received: May 13, 2020; Accepted: May 27, 2020; Published: June 03, 2020

\begin{abstract}
One unique facet of the COVID 19 pandemic is the patient surges that deplete hospitals and hospital systems of critical resources such as equipment, medications, and personnel. Addressing such a surge in patients with coronavirus 2019 (COVID-19) has proven to be challenging in many countries, including in the United States. To prepare for the surge of COVID-19 infected patients in the hospital setting, complex operational preparation plans were enacted with the Kaiser Permanente Southern California Kaiser Health System. Critical care units and emergency departments were identified as venues needing the most assistance. Due to the anticipated cancellation of all elective surgeries, anesthesiologists were identified as an ideal available physician pool for redeployment to these patient care areas. Anesthesiologists have intensive care training in their residencies, and they have expertise performing procedures such as intubation, central line placement, and arterial line placement that are needed to monitor and treat COVID 19 patients. Accordingly, an anesthesiologist led team comprised of physicians and certified nurse anesthetists was developed and named "Sppecial Weapons $\underline{\text { And }}$ Tactics" (SWAT) team. The COVID-19 SWAT response team provided multi-disciplinary clinical consultation, airway management and insertion of invasive catheters for COVID-19 patients in intensive care units, emergency departments, and/or other sectors. The utilization of SWAT teams is one way to maximize operations during a resource strapped event such as the current COVID-19 pandemic and serves as one model to deliver a highly synergistic care delivery for this dynamic and complex pandemic.
\end{abstract}

Keywords: COVID-19, Pandemic, Anesthesiologist, SWAT team, Surge planning

\section{Introduction}

The coronavirus 2019 (COVID-19) pandemic has impacted over 150 countries around the world and has resulted in a global health care crisis [1]. The United States has become one of the most impacted countries and based on most recent CDC data, 12\% of COVID-19 patients require hospitalization and $7 \%$ require admission to intensive care unit (ICU) [2]. Once in the ICU, patients' respiratory status can rapidly deteriorate requiring intubation [3]. The sheer number of COVID-19 patients arriving in the ICU has, in many cases, overwhelmed institutional resources, including those of critical care physician intensivists, nurses (RN) and specialist staff who are already working at maximal levels.

The original SWAT teams were created by Los Angeles Police Department inspector Daryl Gates; he first envisioned "SWAT" as an acronym for "Sppecial Weapons Attack Team" in 1967, but later accepted "Śpecial Weapons $\underline{\text { And }}$ Tactics" on the advice of his deputy chief, Edward M. Davis. Many governmental and health care officials have stated that the COVID-19 pandemic is a war against an invisible enemy being fought by our front-line health care providers. In an effort to provide coordinated assistance, an operational and clinical care plan was devised and implemented to facilitate an anesthesiologist-led "SWAT" team to deliver complex problem-solving capabilities, provide requisite airway management (i.e. intubation), as well as placement of invasive arterial and central venous access for COVID-19 patients in ICUs and Emergency Departments (ED). No subjects were utilized in this clinical innovation, and Institutional Review Board approval was not required.

\section{Clinical Care Innovation}

Delivery of coordinated services in multiple sectors for airway management and placement of invasive catheters by a physicianled anesthesiology SWAT team requires a vast amount of planning that must be facilitated by each respective medical center or temporary medical field hospital through complex infrastructure and logistical operations. Considerations included the pool of available 
anesthesiologists, nurse anesthetists and anesthesiology technologists per day who were not utilized in the operating room for emergent and urgent cases, the number of COVID 19 designated ICU beds in our hospital, and the number of patients undergoing care in the ED. Additionally, surge estimation projections were factored into the manpower and logistics models. Within the southern California Kaiser Permanente Health (KPH) system, every individual Kaiser Permanente hospital ascertained its needs for supplemental physician and healthcare providers based upon suspected or confirmed COVID-19 patient assessment. For our tertiary care hospital integrated in this medical system, the SWAT team was designated the best structure.

SWAT team structure: After a thorough review of the clinical needs in the ICU and ED, the department of anesthesia determined that the anesthesiologist-led SWAT team would be composed of three members: 1) a physician anesthesiologist (MDA) 2) a Certified Registered Nurse Anesthetist (CRNA) or another MDA and 3) an Anesthesiology Technologist (AT). A minimum of one SWAT team was assigned per day based on overall demand for services 24 hoursa-day/7 days-a-week; however, additional teams were added based on surge projection modeling and subsequent opening of more ICUs exclusively designated for the care of COVID-19 patients. In $\mathrm{KPH}$ system, the team was assigned to the entire hospital, but in other hospitals the team (s) could be assigned to a specific sector (i.e. ICU, ED), field hospital or temporary triage area) or work jointly in sequence covering areas as consultations are requested. In the KPH system, SWAT teams were designed as Alpha, Bravo, Charlie, Delta. Each team member was given a cell phone and beeper, and all beepers were synced to deploy simultaneously. Each SWAT team was assigned to one 12-hour shift per day allow for optimal performance without undue fatigue or burn-out of team members.

SWAT team work flow: A major tenet in the SWAT structure is to support each team member to optimize team performance and cohesion through open communication channels. Daily briefing and group "huddles" were coordinated at the beginning and end of every SWAT team shift (i.e. 0700, 1900) and occurred in the anesthesiology workroom. After the 0700 morning huddle, the SWAT team (s) would participate in clinical rounds with the entire ICU staff (physicians, nurses, respiratory therapists, other specialists) to determine which COVID-19 infected patients may likely require interventions, as well as to plan the timing of any necessary procedures by the SWAT team. The evening SWAT team would round with the ICU staff at 2300. For unplanned or emergency services, the ICU intensivist, hospitalist or ED physician would page the team for immediate response.

SWAT teams maintain constant communication to ensure availability, therefore, if one SWAT team is performing a procedure, the other team would be alerted to be readily available immediately to assist in the event of a critical event. Intubation and procedure kits were pre-assembled in "GO BAGS" for immediate "grab and go" functionality. When the SWAT team was called for intubation or procedures, the MDA would communicate directly with the ICU Medical Doctor (MD) to place sedation, ventilator, and restraint orders into Electronic Medical Record (EMR). The SWAT team would communicate with the primary nurse $(\mathrm{RN})$ before starting any procedure (s) so that the $\mathrm{RN}$ could prepare sedation medications or perform additional tasks. The ICU RN should also be in the patient room assisting the SWAT team the entire time during procedural interventions. If time permits, a patient safety briefing (Table 1) with anesthesia SWAT team, RN, and RT was done prior to entering room. Donning and doffing protective equipment was performed with an established spotter - either the CRNA or AT. The spotter must pay close attention during entire procedure and act as a safety agent for both the provider(s) and patient to avoid any breaches in protocol. At the end of procedure(s), close loop with ICU MD, and inform the RN and MD of any issues, medications given or other notable events. At end of shift, get update from ICU MD and sign out to SWAT team MDA.

Table 1: "Time-Out" Safety Check Briefing Checklist.

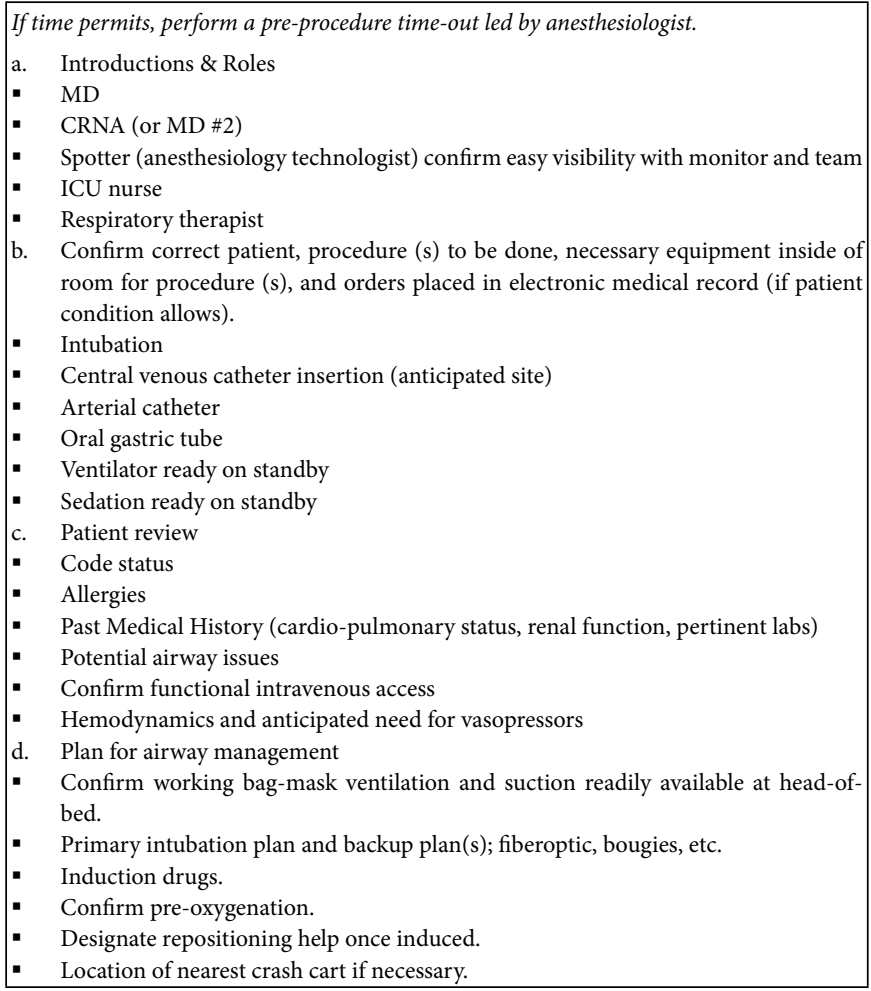

Table 2: SWAT Team Performance Enhancement Synergy.

a. OPEN LOOP COMMUNICATION among all SWAT teams is key.

b. Define and maintain specific roles prior to entering patient room to ensure optimal performance and maximize safety.

c. Always work in groups of 3 anesthesia providers (2 inside room and 1 outside monitoring patient/administering medications)

d. SWAT team(s) should attend rounds in each sector where services for COVID-19 patients will be needed (i.e. ICU, ED)

e. Time procedures to ensure that ancillary support is available, as well as to coincide when patients are in supine position to facilitate procedures.

f. Identify issues for patient care improvement during rounds (i.e. pressure ulcers, face protection, sedation protocols, safety issues) related to perioperative anesthesia expertise.

g. Encourage multi-disciplinary problem solving with other knowledgeable providers (i.e. physician and nursing specialists) from any and all sectors.

h. End each shift with debriefing and close communication channels to ensure planning for next SWAT team.

i. Debriefing with stakeholders from other sectors.

j. Identify any potential deficiencies or obstacles and notify appropriate executive channels for support. 


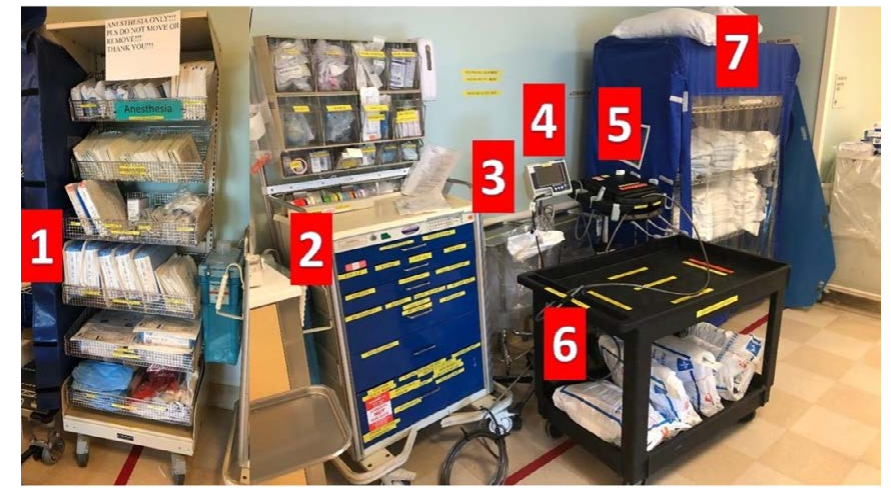

Figure 1: Designated secure area in intensive care unit and emergency department for anesthesia SWAT team supplies. (1) Cart: sterile towels, sterile gloves, central venous catheters (cordis, dialysis catheters); (2) Anesthesia portable cart with full array of medications, syringes, tubing, fluids; ( 3) clean Mayo stand with clear waste bag; (4) Portable video laryngoscope screen (5) Ultrasound machine; (6) Black cart for clean products with "GO BAGS" underneath (7) Linens.

Supply coordination: Due to the need for a wide variety of anesthesiology and procedural supplies, designated areas were partitioned in the various clinical sectors (i.e. ICU, ED). Set-up and inventory control is the responsibility of the anesthesiology technologist. Figure 1 demonstrates the supply set-up area with the necessary supplies. When providing direct care to COVID-19 patients, each team member was equipped with a purified air purifying respirator (PAPR) and full personal protective equipment (PPE). PAPRs were placed in locked orange tackles boxes and designed for each SWAT team member. Designated showers for decontamination at end of shift were also provided for all COVID-19 health care providers.

\section{Discussion}

The COVID-19 pandemic has created a major disruption in day-today care and necessitates emergency coordination of multidisciplinary teams to optimize patient outcome, minimize physician burnout, and improve operations. The use of SWAT teams in medicine have been widely employed to maximize clinical medical mission readiness and effectiveness during disasters [4-7]. The mission of combat/military SWAT is to save lives, and the primary focus of SWAT is to provide tactical solutions that increases the likelihood of de-escalation and safe resolution of high-risk incidents. In the medical setting, we organized our anesthesiologist-led SWAT team around three core concepts. The first was command control -- an anesthesiologist led the team, fielded all consultations, ascertained medical history, ordered pertinent studies pre-procedure as needed, and performed procedures with help of a Certified Nurse Anesthetist (CRNA) and anesthesia technician. The second was containment of risk --the team employed a checklist to ensure appropriate and safe donning and doffing of protective patient equipment PPE with a spotter to ensure actions were performed without self-contamination. The third was rescue - for example, the resuscitation of the patient from respiratory distress via intubation. Therefore, SWAT concepts can be translated and adapted during a pandemic situation that requires multiple layers of operations, logistics and expertise for optimal management [8]. The various steps in the implementation and execution of an anesthesiologist led SWAT team. SWAT team protocols make use of checklists to ensure uniform standards, ensure 24/7 readiness, and maximize provider and patient safety. Additionally, the SWAT team structure can be expanded or tailored based upon a facility's need, size or geographic footprint.

The implementation of an anesthesiologist-led SWAT team reduces the work burden for ICU personnel. As the course of critically ill COVID-19 requires relatively long-term care in the ICU, physician intensivists, nurses, and respiratory therapists may experience burn-out along with their own respective manpower shortages. The anesthesiologist-led SWAT team alleviates the strain of performing complex procedures on critically ill patients. Airway manipulation of patients infected with COVID-19 can be potentially hazardous to any healthcare providers and warrants a high degree of expertise and precautions [9-12]. As airway and invasive monitor experts, an anesthesiologist-led SWAT team employs the principles of in-depth knowledge, training, insight and preparation for duty in highly hazardous clinical settings. The utilization of a SWAT team is a manpower-efficient method of delivering care in resource constrained settings, especially given that a majority of anesthesia providers will have increased availability to due to mandatory cancellation of elective surgical procedures during the COVID-19 pandemic. Appropriate Personal Protective Equipment (PPE) should always follow up-to-date recommendations as set forth by the Centers for Disease Control and Prevention in conjunction with clinical guidelines issued jointly by the American Society of Anesthesiologists, Anesthesia Patient Safety Foundation and American Association of Nurse Anesthetists [2, 13-15].

The fiscal impact of anesthesiologist-led SWAT team implementation is variable and highly dependent upon the overall employment model utilized for anesthesia care providers. In nonsalaried health care provider environments (i.e. private practice "feefor-service'), the fiscal impact of SWAT team costs may be borne out by billing $3^{\text {rd }}$ party payors for procedural relative value units, and/or through hospital stipends derived from state and federal subsidies provided to hospitals for management of COVID-19 patients during a national state of emergency. In academic practices or single- or multispecialty salaried physician groups, the reallocation of health care providers to meaningful work assignments defrays the fixed costs of personnel who would otherwise not have any work to perform. Work performed in distressed situations beyond ordinary duty may possible quality for "hazard pay," and state/federal laws govern this area. SWAT teams enable organized and coordinated provision of critical care services during distressed periods associated with rapidly arising scenario that may last a short or long-term period.

In conclusion, the utilization of an anesthesiologist-led SWAT team is a clinical innovation that is ideally suited to assist during the COVID-19 pandemic. The operational implementation plan described can be utilized in traditional tertiary care medical centers, temporary "field hospitals" or ambulatory surgery centers. In addition to the technical arsenal within our scope of practice, anesthesiologists, nurse anesthetists and anesthesiology technologists bring great insight and problem-solving capabilities to sectors that do not routinely interface with anesthesia providers. Multi-disciplinary teamwork allows a multitude of practitioners to use their expertise to handle a complex pandemic such as COVID-19. 


\section{References}

1. https://www.who.int/emergencies/diseases/novel-coronavirus-2019/situationreports.

2. https://www.cdc.gov/coronavirus/2019-ncov/index.html.

3. Zhou F, Yu T, Du R, Fan G, Liu Y, et al. (2020) Clinical course and risk factors for mortality of adult inpatients with COVID-19 in Wuhan, China: a retrospective cohort study. The Lancet. [Crossref]

4. Heiskell LE, Carmona RH (1994) Tactical emergency medical services: an emerging subspecialty of emergency medicine. Annals of Emergency Medicine 23: 778-785.

5. Jones JS, Reese K, Kenepp G, Krohmer J (1996) Into the fray: integration of emergency medical services and special weapons and tactics (SWAT) teams. Prehospital and Disaster Medicine 11: 202-206. [Crossref]

6. Yeskey KS, Llewellyn CH, Vayer JS (1996) Operational medicine in disasters. Emergency Medicine Clinics 14: 429-438.

7. McArdle DQ, Rasumoff D, Kolman J (1992) Integration of emergency medical services and special weapons and tactics (SWAT) teams: the emergence of the tactically trained medic. Prehospital and Disaster Medicine 7: 285-288.
8. https://post.ca.gov/Portals/0/post_docs/publications/SWAT-2019.pdf.

9. Cheung JC-H, Ho LT, Cheng JV, Cham EYK, Lam KN (2020) Staff safety during emergency airway management for COVID-19 in Hong Kong. The Lancet Respiratory Medicine 8. [Crossref]

10. Brewster DJ, Chrimes NC, Do TB, Fraser K, Groombridge CJ, et al. (2020) Consensus statement: Safe Airway Society principles of airway management and tracheal intubation specific to the COVID-19 adult patient group. The Medical journal of Australia 212: 1. [Crossref]

11. Chen X, Shang Y, Yao S, Liu R, Liu H (2020) Perioperative care provider's considerations in managing patients with the COVID-19 infections. Transl Perioper Pain Med 7: 216-223.

12. Ng K, Poon BH, Puar THK, Quah JLS, Loh WJ, et al. (2020) COVID-19 and the risk to health care workers: a case report. Annals of Internal Medicine. [Crossref]

13. https://www.asahq.org/in-the-spotlight/coronavirus-covid-19-information

14. https://www.apsf.org/covid-19-and-anesthesia-faq/.

15. Learn A (2020) AANA, ASA, APSF and AAAA Issue Joint Statement on Use of Personal Protective Equipment During COVID-19 Pandemic.

\section{Citation:}

Ferrara JT, Kim RY, Gruber JM , Arthur KR, Chen EY, Thuraisingham DA, Melissa Trelatsky CRNA, Lee SJ, Donahue N, Kharestan A, Moreta-Sainz LM, Stranc DS, Rudikoff AG and Conte AH (2020) Operational and clinical implementation plan for an anesthesiologist-led Coronavirus-2019 hospital "SWAT" team. Int J Anes Srg Volume 1(1): 1-4 